\title{
Path Planning of Bio-inspired Swarm of AUVs using Distributed Path Consensus algorithm
}

\author{
Sarada Prasanna Sahoo ${ }^{1}$, Bikramaditya Das $^{2, *}$ and Bibhuti Bhusan Pati ${ }^{1}$ \\ ${ }^{I}$ Department of Electrical Engineering, VSSUT Burla, Odisha, India \\ ${ }^{2}$ Department of Electronics and Telecommunication Engineering, VSSUT Burla, Odisha, India
}

Received 15 June 2021; Accepted 21 November 2021

\begin{abstract}
This paper uses the multi agent system (MAS) as the bioinformatics-inspired technique for guiding a team of autonomous underwater vehicles (AUVs) toward desired destination. This problem mimics the team behaviour towards achieving a common target. Here, each AUV estimates the position of the neighbour AUVs while moving towards the destination. An individual AUV is designated as an agent connected by a communication network and assumes full communication. The proposed multi-AUV system constitutes of a leader AUV and three follower AUVs. A distributed path consensus (DPC) is proposed that determine the distance constraint to ensure the neighbouring agent AUVs must maintain a predefined distance between each other while moving towards the respective destinations. Due to the proposed distance constraint, AUVs stays at a safe distance from each other and also from the static obstacles while maintaining underwater communication using interactive switching topology. The performance of the optimized path is obtained using MATLAB simulation. From the obtained results, it is observed that the proposed control algorithm provides effective co-operative motion control of multiple AUVs along the desired paths and avoid obstacles successively. The proposed method solves coordination problem among multiple AUVs and increase the coverage of underwater missions like oceanographic surveys.
\end{abstract}

Keywords: Autonomous underwater vehicle (AUV), Distance constraint, Distributed path consensus (DPC), Multi-agent system (MAS), Path planning, Position estimation.

\section{Introduction}

The underwater world is vast and to cover a finite area requires involvement of multiple AUVs as a team [1]. The path planning of multiple AUVs using MAS is inspired by team behaviour of humans working towards achieving a common goal within defined time span [2]. The MAS maintains relative positions and orientations deployed as a team while approaching the destination [3]. Control involving path planning needs complicated controller design to maintain inter-vehicular communication to avoid collision among themselves and with other obstacles [4] [5]. The dynamic underwater environment [6] and unavailability of GPS signals [7] make path planning control task quite challenging. Thus, designing fewer complex controllers for path planning control is recently gaining research interest[8], [9]. Most of the methods for path planning control of multiple AUVs advocate leader-follower structure [3-9] where follower vehicles trace the trajectory of a leader AUV. The leader AUV decides the direction of advancement of the formation, while follower AUVs maintain the required angle of orientation and position with respect to the path of the leader [10].

Different consensus algorithms used for the networked multi-agent system (MAS) has been discussed in [11]. The MAS can be considered as solution to coordination problem for multi AUV formation [12]. Szymak et al. [13] developed a control system architecture for MAS consisting AUVs employed in underwater survey. Yang et al. [14] suggested to

*E-mail address: adibik09@gmail.com ISSN: 1791-2377 @ 2021 School of Science, IHU. All rights reserved. doi:10.25103/jestr.145.20 employ Jacobi transform and geometrical reduction techniques to separately design shape, motion and AUV orientation controllers. Xue et al. [15] designed an artificial potential field (APF) based controller for the distributed formation control problem of MAS with varying communication topology. Hu et al. [16] proposed an energy efficient information exchange triggered by impulse signal between AUVs in a multi-AUV formation topology. A formation controller and a consensus law for MAS based on position estimation has been also discussed in literature [13], [17], [15]. A decentralized formation controller for time varying formation topology along with collision avoidance has been proposed in [18]. Liu et al. [19] designed a formation controller using a reference topology and mapping decision for a MAS comprising of identical agents. A distributed consensus observer to estimate the state of a leader for a leader-follower formation topology is discussed in [20].

The efficient path planning of MAS is to determine the path of each agent between user defined start and goal point [21]. An efficient path planning method using Flow Annotation Replanning (FAR) [22] for MAS on grid map proposed by Wang et al. FAR is verified to be scalable, fast, and memory efficient but does not guaranteed completeness. Thus, they proposed a multi-agent path planning (MAPP) [23] algorithm for undirected graphs that ensures completeness with computational efficiency. Bhattacharya et al. [24], [25] developed a distributed path consensus (DPC) algorithm and used it for the path planning and task assignment of MAS. Gaber et al. [26] proposed a PP method by integrating global assignment problem, checkpoint priority action database algorithm and APF method. The decentralized multi-agent Rapidly-exploring Random Tree (DMA-RRT) algorithm has 
been proposed by Desaraju et al. [27] to obtain minimum cost path for MAS. Yu et al. [28] solved MAPP problem by reducing it to network flow problem. He suggested application of network flow algorithms and liner programming to solve the MAPP. Deng et al. [29] proposed a grid-based multi objective optimal programming (GMOOP) path planning method for interacting agents with limited communication ability to execute multi objective tasks. A push and rotate algorithm has also been proposed in [30] to solve MAPP problem. Rossi et.al [31] categorized collective behavioral algorithms of MAS according to their fundamental mathematical structure. A hybrid path planning algorithm for multi-AUVs system has been discussed in [32], where each AUV can be considered as an individual agent. Ma et al. in [33] discussed various issues related MAPP problem in real world scenario. One of the issues to be addressed for MAS is randomly switching communication topology of different agents.

This research is concerned with path planning of coordinated multiple AUVs using switching topology based on the concept of bio-inspired MAS. The bio-inspired MAS is used to handle position of the various AUVs in the underwater environment relative to each other, by considering their position. Here each agent represented by an AUV connected by a communication graph network. The proposed multi-AUV system constitutes of a leader AUV and three follower AUVs. All the AUVs are assumed to be identical. The position estimation method is proposed for estimating the position of neighbour AUV and to plan a path from starting point to desired destination. The distributed path consensus (DPC) is proposed for path optimization by implementing a distance constraint that ensures a predefined distance between the neighbouring AUVs while moving towards the respective destinations. Due to the distance constraint AUVs stays at a safe distance from each other while maintaining communication among the agents. Path optimization is performed for each AUV separately to find a minimum cost route between desired start and destination point.

This research contributions can be listed as follows:

- MAS controller is designed based on the position estimation to obtain a desired shape while approaching towards the destination imposing the distance constraints using DPC algorithm.

- Avoids inter vehicle collision as well as obstacles while prevailing communication and provides optimized path.

- Verification of the performances in terms of tracking error in $\mathrm{X}$ and $\mathrm{Y}$ directions.

\section{Problem formulation}

This research intended to solve a path planning problem [31] using bio-inspired MAS where each agent reach their desired destinations using position estimation technique. The MAS represent a system of identical AUVs deployed for the mission. The AUVs travel from different starting point to different predefined destinations while maintaining a coordinated shape. A MAS controller is designed to obtain the stable shape and to plan paths for agents to reach their destination using position estimation technique. The agents switch from one state to other state with progress in time till the desired shape is obtained. The DPC algorithm is proposed to implement a distance constraint that maintains a safe distance among neighbouring AUVs and helps in avoiding collision among them. The path cost is optimized by using distance constraint for each position of AUV along the path. Once the desired coordinated shape is obtained, the same is maintained by the agent AUVs while approaching their destinations as shown in Figure 1.

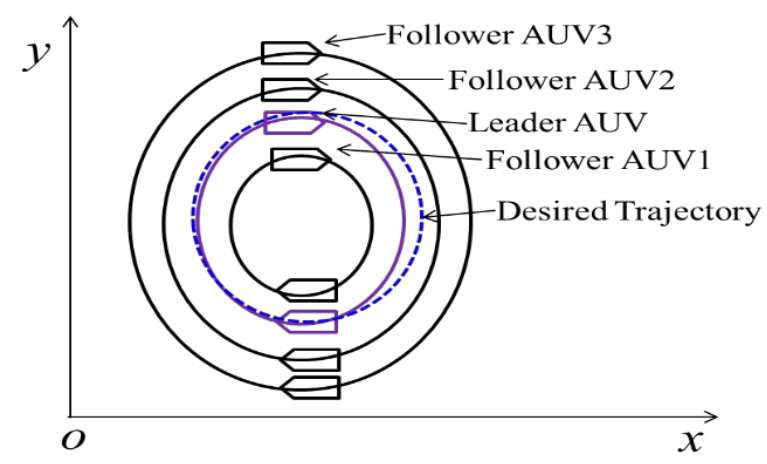

Fig. 1. Schematic presentation of flocking of four AUVs

The DPC algorithm is applied to path of each agent separately to obtain an optimum position to maintain the coordinated shape. At the optimized position the agent AUVs are at a safe distance from the neighbor AUVs without loss of communication among them. It intends to find $\mathrm{K}$ paths for $\mathrm{K}$ agent-AUVs with optimized path cost defined as $\widehat{\mathrm{P}}_{\mathrm{k}}$ for $(1<\mathrm{k}<K)$ through the defined directed graph $\mathcal{G}_{\mathrm{k}}$ such that

$$
\left\{\widehat{\mathrm{P}}_{1}, \ldots, \widehat{\mathrm{P}}_{\mathrm{K}}\right\}=\underset{\widehat{\mathrm{P}}_{1} \ldots, \widehat{\mathrm{P}}_{\mathrm{K}}}{\operatorname{argmin}} \sum_{\mathrm{k}=1 \ldots \mathrm{K}} \mathrm{c}_{\mathcal{E}}\left(\widehat{\mathrm{P}}_{\mathrm{k}}\right)
$$

The equation (1) is subjected to the distance constraint given by

$$
\begin{aligned}
& \mathrm{d}\left(\widehat{\mathrm{P}}_{\mathrm{k}}(\mathrm{t}), \widehat{\mathrm{P}}_{\mathrm{l}}(\mathrm{t})\right) \leq \pi_{\mathrm{kl}}(\mathrm{t}) \forall(1 \leq \mathrm{t} \leq \tau),(1 \leq \mathrm{k} \leq \mathrm{K}), 1 \leq \\
& \mathrm{l} \leq \mathrm{K}, \mathrm{k} \neq \mathrm{l}
\end{aligned}
$$

The environment is modelled as a 2D grid map assuming that all the AUVs are at the same depth. The environment is free of obstacles, Thus, AUVs have to avoid collision among them only.

\section{Communication consensus}

A directed graph $\mathcal{G}_{\mathrm{k}}=(\mathrm{N}, \mathcal{E}, \mathrm{A})$, while $\mathrm{k}=\{1,2, \ldots, \mathrm{K}\}$ with $\mathrm{N}$ set of nodes and $\mathcal{E} \subseteq(\mathrm{N} \times \mathrm{N})$ set of edges can be used to represent an interaction topology of K agent AUVs connected through a communication network. Here, A represents a vector of non negative values such that $A=\left[a_{k l}\right] \in R^{|\varepsilon| \times|\varepsilon|}$ and $|\mathcal{E}|$ is the cardinality of $\mathcal{E}$. The positive $\mathrm{a}_{\mathrm{kl}}$ suggest that $\mathrm{k}^{\text {th }}$ and $\mathrm{l}^{\text {th }}$ AUVs are immediate neighbours with a communication edge otherwise the $\mathrm{a}_{\mathrm{kl}}=0$. The degree of the $\mathrm{k}^{\text {th }}$ node is given by $\mathrm{d}_{\mathrm{k}}=\sum_{\mathrm{l} \in \mathrm{K}_{\mathrm{k}}} \mathrm{a}_{\mathrm{kl}}$. The $\Delta$ is the degree matrix of $\mathcal{G}_{\mathrm{k}}$, which is a $(\mathrm{K} \times \mathrm{K})$ diagonal matrix of $\mathrm{d}_{\mathrm{k}}$. The positive semi-definite Laplacian matrix of $\mathcal{G}_{\mathrm{k}}$ is defined by $\mathrm{L}=\Delta-\mathrm{A}=\left[\ell_{\mathrm{kl}}\right]$, where $\ell_{\mathrm{kl}}$ is

$\ell_{\mathrm{kl}}=\left\{\begin{array}{c}\sum_{\mathrm{l} \in \mathrm{K}_{\mathrm{k}}} \mathrm{a}_{\mathrm{kl}} \forall \mathrm{k}=\mathrm{l} \\ -\mathrm{a}_{\mathrm{kl}} \forall \mathrm{k} \neq \mathrm{l}\end{array}\right.$

The $\mathcal{G}_{\mathrm{k}}$ is formed by ' $\mathrm{i}$ ' subspaces where $1<\mathrm{i}<K$. Target driven path planning of any $\mathrm{k}^{\text {th }}$ agent $\mathrm{AUV}_{\mathrm{k}}$ in a MAS can be described as finding a path with minimum path cost 
through $\mathcal{G}_{\mathrm{k}}$. A time index can be added as an additional variable to $\mathcal{G}_{\mathrm{k}}$ such that $\mathcal{G}_{\text {tk }}=\mathcal{G}_{\mathrm{k}} \times\{0,1,2, \ldots \tau\}$ to obtain time paramertized path [25]. Each node represents a state $\{\mathrm{s}, \mathrm{t}\}$ given by position coordinates of an agent $\mathrm{AUV}\{\mathrm{x}, \mathrm{y}\}$ such that $s \in \mathrm{N}\left(\mathcal{G}_{\mathrm{tk}}\right)$ at a time instant t. An agent AUV can only move from one state $s$ to a next free state $\hat{s}$ along the edge represented by $\{\mathrm{s}, \mathrm{t}\} \rightarrow\{\hat{\mathrm{s}}, \mathrm{t}+1\} \in \mathcal{E}\left(\mathcal{G}_{\mathrm{tk}}\right)$, such that $\widehat{\mathrm{s}} \in$ $\mathcal{E}\left(\mathcal{G}_{\mathrm{k}}\right)$ or $\mathrm{s} \rightarrow \hat{\mathrm{s}} \in \mathcal{E}\left(\mathcal{G}_{\mathrm{k}}\right)$. This condition guarantees only forward movement of AUVs with time. Each edge associated with a positive cost factor $\mathrm{c}_{\mathcal{E}}(\mathrm{s}, \hat{\mathrm{s}})$. The cost is represented by the Euclidian distances between the neighbouring cell centres. Each path from start to destination point assumed to be accomplished in $\tau$ - time-steps. Thus, the path of $\mathrm{k}^{\text {th }}$ AUV $\left(\mathrm{AUV}_{\mathrm{k}}\right)$ can be given as $\mathrm{P}_{\mathrm{k}}=\left\{\operatorname{start}_{\mathrm{k}}, \mathrm{s}_{1}, \ldots \ldots, \operatorname{target}_{\mathrm{k}}\right\}$. The path cost of path $\mathrm{P}_{\mathrm{k}}$ can be defined as

$\mathrm{c}_{\mathcal{E}}\left(\mathrm{P}_{\mathrm{k}}\right)=\sum_{\mathrm{i}=1 \ldots \tau} \mathrm{c}_{\mathcal{E}}\left(\mathrm{s}_{\mathrm{i}-1}, \mathrm{~s}_{\mathrm{i}}\right)$

The maximum Euclidian distance between a pair of neighbour AUVs has been considered without breaking communication between them as a constraint $\pi_{\mathrm{kl}}$ for all $\mathrm{k} \neq$ 1. The distance between $\mathrm{k}^{\text {th }}$ and $\mathrm{l}^{\text {th }}$ AUVs is defined as $d(\mathrm{~s}, \hat{\mathrm{s}})$ is a positive scalar quantity for any pair of states such that $\mathrm{s} \in \mathrm{N}\left(\mathcal{G}_{\mathrm{k}}\right), \hat{\mathrm{s}} \in \mathrm{N}\left(\mathcal{G}_{\mathrm{l}}\right)$ for $\mathrm{k} \neq \mathrm{l}$. Hence, $\pi_{\mathrm{kl}}(\mathrm{t})$ can be defined as a vector consisting of $\tau$ positive distance values $d(.,$.$) between \mathrm{k}^{\text {th }}$ and $\mathrm{l}^{\text {th }}$ AUVs at time instant $\mathrm{t}$.

The coordination among AUVs results in rendezvous in space [11] consensus problem, where $\mathrm{K}$ agent AUVs reach a consensus with an interactive switching topology through position estimation. The AUV system dynamics can be approximated as a second order liner system as follows:

$\left\{\begin{array}{l}\dot{\eta}_{\mathrm{k}}=\mathrm{V}_{\mathrm{k}} \\ \dot{V_{\mathrm{k}}}=\mathrm{T}_{\mathrm{k}}\end{array}\right.$

where, $V_{k} \in R^{k}, \eta_{k} \in R^{k}, T_{k} \in R^{k}$. In real world problem it is difficult to find exact position $\eta_{k}$ of the $\mathrm{k}^{\text {th }}$ agent AUV. So the following assumptions are made as per [34]:

i. Each $\mathrm{k}^{\text {th }}$ agent AUV estimate the relative position of the neighboring $\mathrm{l}^{\text {th }}$ AUV as it is unable get the exact position. Thus, the relative position of $\mathrm{l}^{\text {th }}$ AUV to $\mathrm{k}^{\text {th }}$ AUV can be calculated as

$$
\eta_{\mathrm{lk}}=\eta_{\mathrm{l}}-\eta_{\mathrm{k}} \forall \forall(1 \leq \mathrm{k} \leq \mathrm{K}), 1 \leq \mathrm{l} \leq \mathrm{K}, \mathrm{k} \neq \mathrm{l}
$$

ii. Each AUV is able to measure its own actual velocity and can also obtain its neighbor velocity information through communication network.

iii. The $\mathrm{k}^{\text {th }}$ agent AUV can estimate position of itself as $\hat{\eta}_{\mathrm{k}}$ for $\mathrm{k}=1, \ldots, \mathrm{K}$ and also able to obtain the position estimation of its neighbour AUV within its communication range defined by the distance constraint $\pi_{i j}(t)$.

iv. The environment is free of static obstacle. Thus, the AUVs themselves are act as dynamic obstacle and avoid collision among themselves by position estimation.

Now the desired position of an agent AUV in the interactive graph $\mathcal{G}_{\mathrm{k}}$ can be defined as $\eta_{\mathrm{kd}}$ where $\mathrm{k}=$ $\{1,2, \ldots, \mathrm{K}\}$.

Lemma $\quad 1: \quad$ For
$\gamma \mu-\beta \pm \sqrt{(\gamma h-\beta)^{2}+4 \mu} / 2$ any where $\sigma, \mu \in C$.
If $\beta \geq 0, \operatorname{Re}(\mu)<0, \operatorname{Im}(\mu)>0$ and $\zeta(\mu)=$ $\sqrt{2 /|\mu| \cos \left[\tan ^{-1} \frac{\operatorname{Im}(\mu)}{-\operatorname{Re}(\mu)}\right\rfloor}$ then $\operatorname{Re}(\sigma)<0$. Here, $\operatorname{Re}($.$) and$ $\operatorname{Im}($.$) are the real and imaginary parts of a number$ respectively. For such condition

$\gamma>\zeta(\mu)$

Lemma 2: For the system given by equation (5) we can use a control law $T_{\mathrm{kl}}$ as given in [34], such that

$\mathrm{T}_{\mathrm{k}}=\sum_{\mathrm{l}=1}^{\mathrm{K}} \mathrm{a}_{\mathrm{kl}}(\mathrm{t})\left[\left(\eta_{\mathrm{k}}-\eta_{\mathrm{l}}\right)+\gamma\left(\mathrm{V}_{\mathrm{k}}-\mathrm{V}_{\mathrm{l}}\right)\right]$

The MAS of AUVs obtains consensus asymptotically if and only if $\partial=\left[\begin{array}{cc}0_{K \times K} & I_{K} \\ -L_{K}(t) & \gamma L_{K}(t)\end{array}\right]$ has two eigen values equal to ' 0 ' and restwith negative real parts. Here, $\eta_{k}(t) \rightarrow$ $\sum_{\mathrm{k}=1}^{\mathrm{K}} \eta_{\mathrm{k}} \eta_{\mathrm{k}}(0)+\sum_{\mathrm{k}=1}^{\mathrm{K}} \eta_{\mathrm{k}} \mathrm{V}_{\mathrm{k}}(0)$ and $\mathrm{V}_{\mathrm{k}}(\mathrm{t}) \rightarrow \sum_{\mathrm{k}=1}^{\mathrm{K}} \eta_{\mathrm{k}} \mathrm{V}_{\mathrm{k}}(0)$ for larger value of $t$, where $\eta=\left[\eta_{1}, \ldots, \eta_{k}\right]^{T} \geq 0, I_{K}{ }^{T} \eta=$ $1, \mathrm{~L}_{\mathrm{K}}^{\mathrm{T}} \eta=0$.

Lemma 3: Assuming that directed graph $\mathcal{G}_{\mathrm{k}}$ has a spanning tree and Laplacian matrix given by $\mathrm{L}, \eta_{\mathrm{k}}=\left\{\eta_{1}, \eta_{2}, \ldots, \eta_{\mathrm{K}}\right\} \in$ $\mathrm{R}^{\mathrm{K}}$ of the linear system given in equation (5) exponentially converges to a finite vector $\eta_{\mathrm{k}}^{\infty}=\left\{\eta_{\mathrm{c}}^{\infty}, \ldots, \eta_{\mathrm{c}}^{\infty}\right\} \in \mathrm{R}^{\mathrm{K}}$ [34].

The formation controller for Multi-AUV system designed to achieves following objectives:

i.To design an estimation law for an $\mathrm{k}^{\text {th }}$ agent-AUV that satisfy

$\lim _{t \rightarrow \infty} \hat{\eta}_{k}(t)=\eta_{k}(t)+\widetilde{\eta_{k}^{\infty}}$

where $\eta_{\mathrm{k}}^{\infty} \in \mathrm{R}^{\mathrm{K}}$ is a constant vector by using $\eta_{\mathrm{kl}}$ and $\hat{\eta}_{\mathrm{l}} \forall \mathrm{l} \in \mathrm{K}_{\mathrm{k}}$. As per equation (9) the estimated position $\hat{\eta}_{\mathrm{k}}$ instead of converging to $\eta_{\mathrm{k}}$ converges to $\eta_{\mathrm{k}}+\overline{\eta_{\mathrm{k}}^{\infty}}$.

ii.To formulate an optimal control law for a set of desired positions of AUV $\eta_{\mathrm{kd}}, \mathrm{k} \in \mathrm{N}$ as per estimator designed such that all the agent AUV reach their respective desired positions with a constant error given by,

iii.

$\left\{\begin{array}{c}\lim _{t \rightarrow \infty} \hat{\eta}_{k}(t)=\eta_{k d}(t)+\widetilde{\eta_{k}^{\infty}} \\ \lim _{t \rightarrow \infty} V_{k}(t)=0\end{array}\right.$ for $k \in\{1,2, \ldots, K\}$

where $\eta_{\mathrm{k}}^{\infty} \in \mathrm{R}^{\mathrm{K}}$ is a constant vector. The energy cost is defined by the quadratic performance index [34] as follows:

$\mathbb{J}=\frac{1}{2} \sum_{\mathrm{k}=1}^{\mathrm{K}}\left\{\int_{0}^{\infty}\left[\mathrm{e}_{\mathrm{k}}(\mathrm{t})^{\mathrm{T}} \mathrm{Q} \mathrm{e}_{\mathrm{k}}(\mathrm{t})+\mathrm{T}_{\mathrm{k}}^{\mathrm{T}} \mathrm{F} \mathrm{T}_{\mathrm{k}}\right] \mathrm{dt}\right\}$

Here, $e_{k}(t)=\eta_{k d}(t)-\hat{\eta}_{k}(t)$ gives the error in output, $\mathrm{Q}$ and $\mathrm{F}$ are assumed to be positive definite matrix of required dimensions. As per equation (10) the estimated position $\hat{\eta}_{\mathrm{k}}$ instead of converging to desired position $\eta_{\mathrm{kd}}$ converges to $\eta_{k d}+\overline{\eta_{k}^{\infty}}$, because of the constant error $e_{k}(t)$. iv.To propose a consensus law as per estimator designed for a set of agent AUVs, so as to make them converge to a consistent value of position and velocity, such that 
$\left\{\begin{array}{c}\lim _{t \rightarrow \infty} \eta_{k}(t)=\lim _{t \rightarrow \infty} \eta_{l}(t) \\ \lim _{t \rightarrow \infty} V_{k}(t)=\lim _{t \rightarrow \infty} V_{l}(t)=\text { constant }\end{array} \forall k, l \in\{1,2, \ldots, K\}(12)\right.$

The final velocities of all the agents as per equation (11) converge to a constant value determined by the initial velocities of all the agent AUVs.

In this research, the available data is obtained only through communication network. The relative position of $\mathrm{k}^{\text {th }}$ and $\mathrm{l}^{\text {th }}$ neighbor AUVs $\left(\eta_{\mathrm{kl}}\right)$, the estimated positions of a pair of neighbour AUV $\left(\hat{\eta}_{\mathrm{k}}, \hat{\eta}_{\mathrm{l}}\right)$ and the velocity measurement $\mathrm{V}_{\mathrm{k}}$ are calculated based on received information.

\section{Controller design}

\subsection{Proposed position Estimator}

For agent AUVs with dynamics given in equation (5) a distributed estimation law [34] can be designed as follows:

$\hat{\hat{\eta}_{\mathrm{k}}}=\mathrm{V}_{\mathrm{k}}+\mathrm{p}_{0} \sum_{\mathrm{l}=1}^{\mathrm{K}} \mathrm{a}_{\mathrm{kl}}\left(\hat{\eta}_{\mathrm{lk}}-\eta_{\mathrm{lk}}\right)=\dot{\eta_{\mathrm{k}}}+\mathrm{o}_{0} \sum_{\mathrm{l}=1}^{\mathrm{K}} \mathrm{a}_{\mathrm{kl}}\left[\left(\hat{\eta}_{\mathrm{l}}-\right.\right.$ $\left.\left.\hat{\eta}_{\mathrm{k}}\right)-\left(\eta_{\mathrm{l}}-\eta_{\mathrm{k}}\right)\right]$

Where, $\mathbb{1}_{0}$ is a positive real number, the estimation of relative position of lth and kth AUV is $\hat{\eta}_{\mathrm{lk}}$. The required velocity $v_{k}$ is calculated. The information about relative position $\left(\eta_{\mathrm{lk}}\right)$ and estimation of position of lth AUV $\left(\hat{\eta}_{\mathrm{l}}\right)$ can be obtained through communication network. If $\tilde{\eta}_{k}=\eta_{k}-$ $\hat{\eta}_{\mathrm{k}}$, then equation (13) can be rewritten as follows:

$\tilde{\eta}_{\mathrm{k}}=\stackrel{\mathrm{n}}{0}_{0} \sum_{\mathrm{l}=1}^{\mathrm{K}} \mathrm{a}_{\mathrm{kl}}\left(\tilde{\eta}_{\mathrm{l}}-\tilde{\eta}_{\mathrm{k}}\right)$

Thus, we can estimate the error dynamics of the overall MAS as Kronicher product of Laplacian matrix L and identity matrix $I(L \otimes I)$ such that;

$\dot{\tilde{\eta}}=-\mathbb{1}_{0}(L \otimes I) \tilde{\eta}$

where, $\tilde{\eta}=\left\{\tilde{\eta}_{1}, \ldots, \tilde{\eta}_{K}\right\}$.

Lemma 4: Assuming that directed graph $\mathcal{G}_{\mathrm{k}}$ has a spanning tree, the estimation of error dynamics $\hat{\eta}_{\mathrm{k}}$ can globally exponentially converges to $\tilde{\eta}-\tilde{\eta}_{\mathrm{k}}{ }^{\infty}$, where $\tilde{\eta}_{\mathrm{k}}{ }^{\infty}=$ $\left\{\eta_{\mathrm{c}}^{\infty}, \ldots, \eta_{\mathrm{c}}^{\infty}\right\}$ is a finite vector $\eta_{\mathrm{k}}^{\infty}=\left\{\eta_{\mathrm{c}}^{\infty}, \ldots, \eta_{\mathrm{c}}^{\infty}\right\}[34]$.

\subsection{Derivation of control input based on consensus law} As per the above assumption and position estimator design, we can define the feedback control law for MAS of AUVs as:

$T_{k}=\sum_{l=1}^{K} a_{k l}\left[\left(\hat{\eta}_{k}-\eta_{k d}\right)-\left(\hat{\eta}_{l}-\eta_{l d}\right)+\gamma\left(V_{k}-V_{l}\right)\right]$

Using equation (12), the desired formation of MAS may be obtained if, $\hat{\eta}_{k} \rightarrow \eta_{k d}$ and $\hat{\eta}_{l} \rightarrow \eta_{l d}$ for all $k, l \in$ $\{1,2, \ldots, K\}$ as $\left\|\hat{\eta}_{\mathrm{k}}-\eta_{\mathrm{kd}}\right\|-\left\|\hat{\eta}_{\mathrm{l}}-\eta_{\mathrm{ld}}\right\| \rightarrow 0$ and $\| \mathrm{V}_{\mathrm{k}}-$ $\mathrm{V}_{\mathrm{l}} \| \rightarrow 0$.

When converging to desired position the agent AUVs should not collide with each other. Thus, distance constraint should be incorporate with the formation controller. Implementation of the distance constraint needs controller design with extra control terms. The potential function is design as per the position estimator as follows:

$\mathbb{V}_{\mathrm{kl}}\left(\hat{\eta}_{\mathrm{k}}, \hat{\eta}_{\mathrm{l}}\right)=\left(\min \left\{0, \frac{\left\|\hat{\eta}_{\mathrm{k}}-\widehat{\eta}_{1}\right\|^{2}-\Re^{2}}{\left\|\hat{\eta}_{\mathrm{k}}-\widehat{\eta}_{\mathrm{l}}\right\|^{2}-\mathbb{r}^{2}}\right\}\right)^{2}$
Here $\Re$ and $\mathbb{r}$ defines the detection radius of the AUV and the safe distance to avoid inter-vehicle collision respectively. If the inter vehicle distance between agent AUVs are less then $\mathfrak{R}$, then $\mathbb{V}_{\mathrm{kl}}\left(\hat{\eta}_{\mathrm{k}}, \hat{\eta}_{\mathrm{l}}\right)$ is positive and will be included in additional control input. By finding the partial derivative of equation (17) with respect to $\hat{\eta}_{\mathrm{k}}$ we can get

$$
\begin{aligned}
& \frac{\partial \mathbb{V}_{\mathrm{kl}}^{\mathrm{T}}}{\partial \widehat{\eta}_{\mathrm{k}}}=\left\{\begin{array}{l}
\frac{4\left(\Re^{2}-\mathrm{r}^{2}\right)\left(\left\|\hat{\eta}_{\mathrm{k}}-\widehat{\eta}_{\mathrm{l}}\right\|^{2}-\Re^{2}\right)}{\left(\left\|\hat{\eta}_{\mathrm{k}}-\widehat{\eta}_{\mathrm{l}}\right\|^{2}-\mathrm{r}^{2}\right)^{3}} \\
0, \text { for } \Re>\left\|\hat{\eta}_{\mathrm{k}}-\hat{\eta}_{\mathrm{l}}\right\|
\end{array}\left(\hat{\eta}_{\mathrm{k}}-\hat{\eta}_{\mathrm{l}}\right)^{\mathrm{T}}, \text { for } \mathbb{r} \leq \| \hat{\eta}_{\mathrm{k}}-\right. \\
& \left.\hat{\eta}_{\mathrm{l}} \| \leq \Re\right\}
\end{aligned}
$$

Now the control law for formation control with collision avoidance based on position estimation can be defined using equation (16) and equation (18) as follows:

$$
\begin{aligned}
& \mathrm{T}_{\mathrm{k}}^{\mathrm{a}}=-\sum_{\mathrm{l}=1}^{\mathrm{K}} \mathrm{a}_{\mathrm{kl}}\left[\left(\left(\hat{\eta}_{\mathrm{k}}-\eta_{\mathrm{kd}}\right)-\left(\hat{\eta}_{\mathrm{l}}-\eta_{\mathrm{ld}}\right)\right)+\gamma\left(\mathrm{V}_{\mathrm{k}}-\right.\right. \\
& \left.\left.\mathrm{V}_{\mathrm{l}}\right)\right]-\sum_{\mathrm{l}=1}^{\mathrm{K}} \frac{\partial \mathbb{V}_{\mathrm{kl}}\left(\widehat{\eta}_{\mathrm{k}}, \widehat{\eta}_{\mathrm{l}}\right)^{\mathrm{T}}}{\partial \widehat{\eta}_{\mathrm{k}}}
\end{aligned}
$$

Theorem 1- As per equation (8) and equation (19) the MAS given in equation (5) obtained the obtained the desirer formation if the directed graph $\mathcal{G}_{\mathrm{k}}$ has a spanning tree, if

$\gamma>\bar{\gamma}$

where $\gamma \triangleq 0$ if all the (K-1) nonzero Eigen value of $-\mathrm{L}_{\mathrm{n}}$, given by $\mu_{\mathrm{k}} \forall \mathrm{k} \in\{1, \ldots, \mathrm{K}\}$ are negative and $\bar{\gamma}_{\max \mu_{k}, \forall k \in\{1, \ldots, K\}} \zeta\left(\mu_{k}\right)$. Therefore , for $t \rightarrow \infty$, we have $\eta_{\mathrm{k}}(\mathrm{t}) \rightarrow \eta_{\mathrm{kd}}+\tilde{\eta}_{\mathrm{c}}^{\infty}+\mathrm{t} \sum_{\mathrm{k}=1}^{\mathrm{K}} \eta_{\mathrm{k}} \mathrm{V}_{\mathrm{k}}(0) \quad$ and $\quad \mathrm{V}_{\mathrm{k}}(\mathrm{t}) \rightarrow$ $\sum_{\mathrm{k}=1}^{\mathrm{K}} \eta_{\mathrm{k}} \mathrm{V}_{\mathrm{k}}(0) \quad$ where $\quad \eta=\left[\eta_{1}, \ldots, \eta_{\mathrm{k}}\right]^{\mathrm{T}} \geq 0, \mathrm{I}_{\mathrm{K}}^{\mathrm{T}} \eta=$ $1, \mathrm{~L}_{\mathrm{K}}^{\mathrm{T}} \eta=1, \tilde{\eta}_{\mathrm{k}}^{\infty}=\left\{\tilde{\eta}_{\mathrm{c}}^{\infty}, \ldots, \tilde{\eta}_{\mathrm{c}}^{\infty}\right\}$ and $\quad \eta_{\mathrm{kd}}=$ $\left\{\eta_{1 \mathrm{~d}}, \eta_{2 \mathrm{~d}}, \ldots, \eta_{\mathrm{Kd}}\right\}$ are constants [34].

Theorem 2- If the controller is designed as per the equation (19) for the system described in equation (5) and $\gamma$ satisfies equation (20), then multi-AUV system obtain its desired formation without inter-vehicular collision [34]. For the proof of theorem 1 and 2, we suggest readers to refer [34].

\subsection{Distributed path consesus (DPC) algorithm}

In this research work, the DPC algorithm is used for planning a safe path for the AUVs to reach the desired destination by applying distance constrant in a 2D underwater environment. Path planning is incorporated for each agent separately using DPC to find K optimal safe paths for K agent-AUVs defined as $\hat{P}_{k}$ for $(1<k<K)$ through the defined directed graph $\mathcal{G}_{k}$ as defined in equation (1) subjected to constraints defined in equation (2). For every iteration iter, the DPC algorithm searches a subset of graph $\mathcal{G}_{\text {sub }}$ to find a path with optimized path cost for an agent AUV [24]. The path cost is the summation of distance travelled by AUV and the penalty imposed due to violation of distance constrained. The penalty for violation increases with each iteration by slowly increasing the weights associated with the cross points. The cross points are the points on the planned path where two paths may collide with each other. Thus, the path cost of generated paths that goes through the cross points increase due to the violation of distance constrained. Hence, these paths are avoided while planning optimized path. The penalty function $\lambda_{\mathrm{kl}}\left(\widehat{\mathrm{P}}_{\mathrm{k}}, \widehat{\mathrm{P}}_{\mathrm{l}}, \pi_{\mathrm{kl}}\right), \mathrm{k} \neq \mathrm{l}$ used for violation of constraint is given by 
$\lambda_{\mathrm{kl}}\left(\widehat{\mathrm{P}}_{\mathrm{k}}, \widehat{\mathrm{P}}_{\mathrm{l}}, \pi_{\mathrm{kl}}\right)=\sum_{\mathrm{t}=1,2, \ldots \tau} \widetilde{\mathrm{w}_{\mathrm{kl}}}\left(\widehat{\mathrm{P}}_{\mathrm{k}}(\mathrm{t}), \widehat{\mathrm{P}}_{\mathrm{l}}(\mathrm{t}), \pi_{\mathrm{kl}}(\mathrm{t})\right)$

If $w_{\mathrm{kl}}$ is the dynamic weight associated with the edge $\mathrm{s} \rightarrow \hat{\mathrm{s}} \in \mathcal{E}\left(\mathcal{G}_{\mathrm{k}}\right)$ such that $\widetilde{w}_{\mathrm{kl}}=w_{\mathrm{lk}}$ then $\widetilde{w_{\mathrm{kl}}}$ can be defined as

$w_{\mathrm{kl}}\left(\mathrm{s}, \hat{\mathrm{s}}, \pi_{\mathrm{kl}}\right)=\max \left(0, \mathrm{~d}(\mathrm{~s}, \hat{\mathrm{s}})-\pi_{\mathrm{kl}}\right)$

As $\pi_{\mathrm{kl}}$ is a constant for our research problem, it can be dropped. Hence, $\lambda_{\mathrm{kl}}\left(\widehat{\mathrm{P}}_{\mathrm{k}}, \widehat{\mathrm{P}}_{\mathrm{P}}, \pi_{\mathrm{kl}}\right)$ is represented as $\lambda\left(\widehat{\mathrm{P}}_{\mathrm{k}}, \widehat{\mathrm{P}}_{\mathrm{l}}\right)$. The association of $w_{\mathrm{kl}}$ with $\pi_{\mathrm{kl}}$ is to convert the hard distance constraint defined in equation (2) to a soft distance constraint [24]. The path cost is computed by calculating the Euclidian distance between the AUVs by using position estimation. Then, DPC algorithm is used iteratively on each agent AUV to optimize the cost. For $\mathrm{k}^{\text {th }}$ agent AUV, the weight of penalty increases by a small amount $\mho_{\mathrm{kl}}^{\mathrm{i}}$ within each iteration. The value of $\mho_{\mathrm{kl}}^{\mathrm{i}}$ is calculated as

$\mho_{\mathrm{kl}}^{\mathrm{i}}=\left\{\begin{array}{l}\geq 0, \forall \mathrm{k} \neq 1 \\ 0, \text { otherwise }\end{array}\right.$

Thus, a minimum cost path is computed for $\mathrm{k}^{\text {th }} \mathrm{AUV}$ can be given as $\mathrm{P}_{\mathrm{k}}=\left\{\operatorname{start}_{\mathrm{k}}, \mathrm{s}_{1}, \ldots \ldots\right.$, target $\left._{\mathrm{k}}\right\}$ in $\mathcal{G}_{\mathrm{tk}}$. The cost associated with the transition $\{\mathrm{s}, \mathrm{t}\} \rightarrow\{\hat{\mathrm{s}}, \mathrm{t}+1\} \in$ $\mathcal{E}\left(\mathcal{G}_{\mathrm{tk}}\right)$ has been modified using

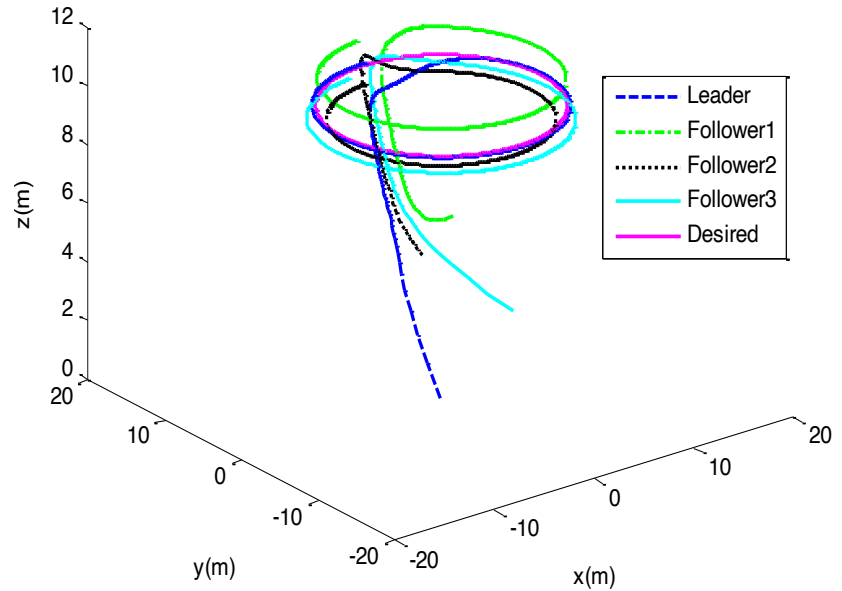

(a)
$\mathrm{c}_{\mathcal{E}}(\mathrm{s}, \widehat{\mathrm{s}})+2 \sum_{\mathrm{l}=1 \ldots \mathrm{K}, \forall \mathrm{k} \neq 1} w_{\mathrm{kl}}^{\mathrm{iter}+1} \lambda_{\mathrm{kl}}\left(\widehat{\mathrm{s}}, \widehat{\mathrm{P}}_{\mathrm{l}}^{\mathrm{iter}}(\mathrm{t}), \pi_{\mathrm{kl}}(\mathrm{t})\right)$

For, each iteration iter and $\mathrm{i}^{\text {th }}$ AUV path can be calculated as

$$
\begin{aligned}
& \widehat{\mathrm{P}}_{\mathrm{i}}^{i t e r}
\end{aligned}
$$

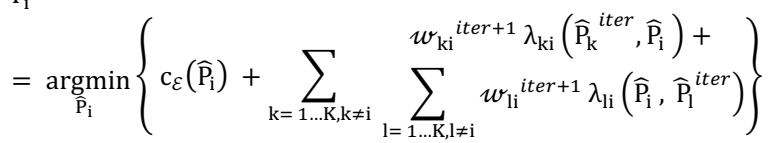

$$
\begin{aligned}
& =\underset{\widehat{\mathrm{P}}_{\mathrm{i}}}{\operatorname{argmin}}\left\{\mathrm{c}_{\mathcal{E}}\left(\widehat{\mathrm{P}}_{\mathrm{i}}\right)+\right. \\
& \left.2 \sum_{\mathrm{l}=1 \ldots \mathrm{K}, \forall \mathrm{k} \neq \mathrm{i}} w_{\mathrm{ki}}{ }^{i \text { ter }+1} \lambda_{\mathrm{ki}}\left(\widehat{\mathrm{P}}_{\mathrm{k}}{ }^{i \text { ter }}, \widehat{\mathrm{P}}_{\mathrm{i}}\right)\right\}
\end{aligned}
$$

\section{Result Analysis}

The proposed path planner for MAS of multiple AUVs has been tested by simulation performed using MATLAB. This simulation shows a leader AUV and three follower AUVs. The follower AUVs are represented as AUV1, AUV2 and AUV3. The simulation parameters for the AUV are taken from the experimental values as in [35], [36].

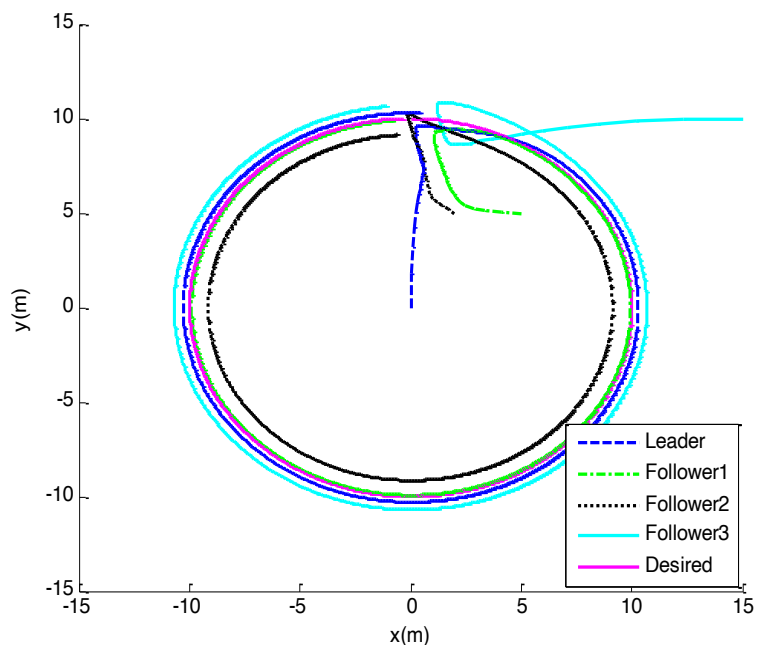

(b)

Fig. 2. Path planning of Bio-inspired AUVs in circular path avoiding inter vehicle collision (a) 3D space (b) 2D plane

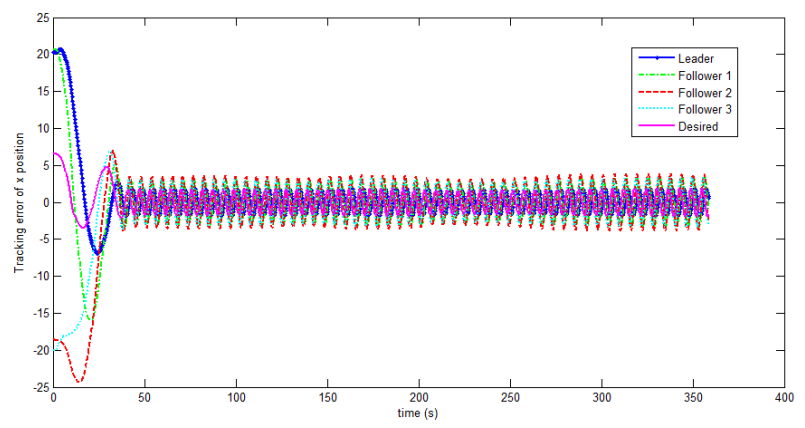

Fig. 3. Tracking error in X-direction

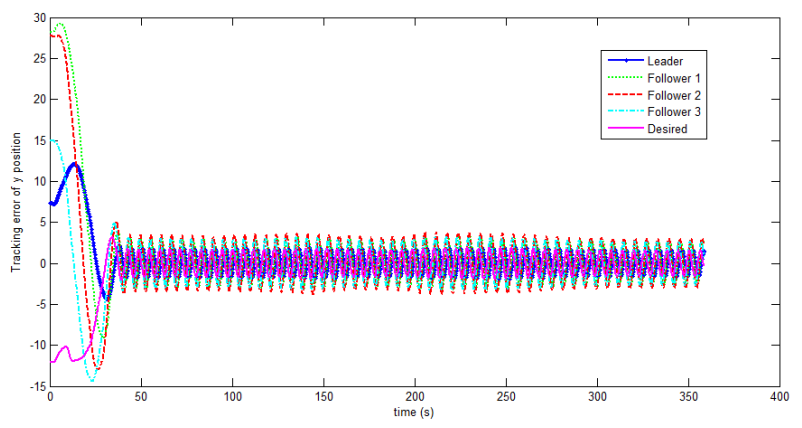

Fig. 4. Tracking error in Y-direction

Table 1. Variation of tracking error in $\mathrm{X}$ and $\mathrm{Y}$ direction

\begin{tabular}{c|c|c|c|c|c|c|c|c|c|c|c|c}
\hline Time (sec) & \multicolumn{2}{|c|}{$\mathbf{0}$} & \multicolumn{2}{c|}{$\mathbf{1 0}$} & \multicolumn{2}{c|}{$\mathbf{2 0}$} & \multicolumn{2}{c|}{$\mathbf{3 0}$} & \multicolumn{2}{c|}{$\mathbf{4 0}$} & \multicolumn{2}{c}{$\mathbf{5 0}$} \\
\hline Tracking error (m) & $\mathbf{X}$ & $\mathbf{Y}$ & $\mathbf{X}$ & $\mathbf{Y}$ & $\mathbf{X}$ & $\mathbf{Y}$ & $\mathbf{X}$ & $\mathbf{Y}$ & $\mathbf{X}$ & $\mathbf{Y}$ & $\mathbf{X}$ & $\mathbf{Y}$ \\
\hline Leader AUV & 21 & 7 & 15 & 11 & -4 & 8 & -3 & -4 & -2 & 0.1 & 1.3 & 1.5 \\
Follower 1 & 20 & 28 & 6 & 27 & -16 & 7 & -1 & -9 & -2.5 & -1.5 & 1 & 3 \\
Follower 2 & -19 & 27.7 & -22 & 23 & -19 & -4 & 4 & -10 & -2 & -3 & -0.6 & 3.5 \\
Follower 3 & -20 & 15 & -18 & 7.5 & -10 & -12.5 & 7 & -6 & -0.8 & -3.5 & -2 & 2.5 \\
\hline
\end{tabular}


From the Figure 2 (a) to Figure 2(b) show the positions obtained by all the AUVs at different $3 \mathrm{D}$ and $2 \mathrm{D}$ domain respectively. Figure 2 show that all the follower AUVs follow the desired path by the leader AUV avoiding collision among themselves. Figure 3 and Figure 4 show the tracking error variations from 0 to 350 second in $\mathrm{X}$ direction and in $\mathrm{Y}$

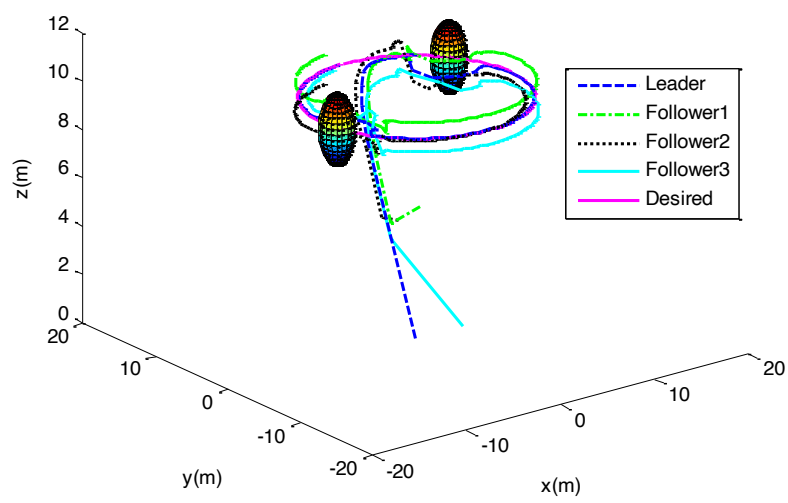

(a) direction respectively. From the Table 1, it is observed that as the follower AUV start to follow the desired path of the leader AUV in $\mathrm{X}$ and $\mathrm{Y}$ direction in the time period 0 to $50 \mathrm{sec}$. After $30 \mathrm{sec}$, it can also be observed that follower AUVs do not track the leader AUV but try to follow the path of the leader AUV.

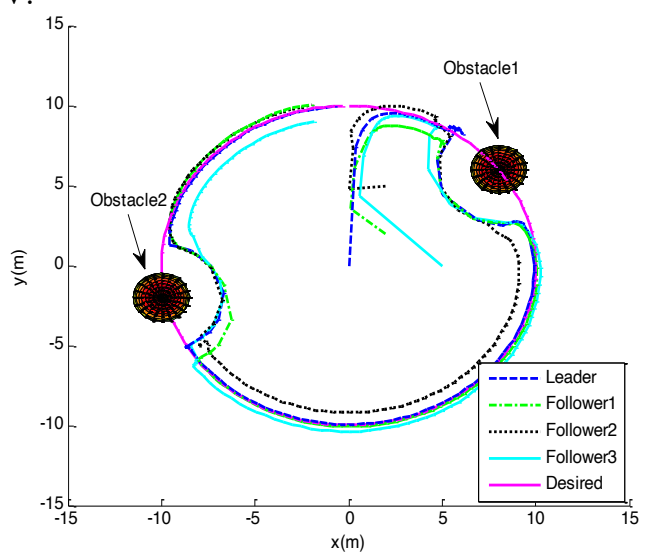

(b)

Fig. 5. Path planning of Bio-inspired AUVs in circular path and avoiding solid obstacles as well as inter vehicle communication in (a) 3D space (b) 2D plane

From Figure 5, it is observed that there is no chance of collision among AUVs or AUV with obstacles arisen during path planning.

Table 2. Comparisons of flocking approach times and distances among AUVs for both the methods

\begin{tabular}{|c|c|c|c|c|c|c|}
\hline \multirow{3}{*}{$\begin{array}{c}\text { Control } \\
\text { strategy for } \\
\text { different cases }\end{array}$} & \multicolumn{3}{|c|}{ Inter vehicular avoidance } & \multicolumn{3}{|c|}{$\begin{array}{c}\text { Inter vehicular avoidance as well as static } \\
\text { obstacle avoidance }\end{array}$} \\
\hline & \multirow{2}{*}{$\begin{array}{c}\text { Path } \\
\text { planning } \\
\text { approach } \\
\text { time (sec) }\end{array}$} & \multicolumn{2}{|c|}{ Distance between AUVs (m) } & \multirow{2}{*}{$\begin{array}{c}\text { Path } \\
\text { planning } \\
\text { approach } \\
\text { time (sec) }\end{array}$} & \multicolumn{2}{|c|}{$\begin{array}{c}\text { Distance between } \\
\text { AUVs (m) }\end{array}$} \\
\hline & & Minimum & Maximum & & Minimum & Maximum \\
\hline $\begin{array}{l}\text { One leader and } \\
\text { three followers }\end{array}$ & 2.4 & 1.584 & 1.611 & 1.2 & 1.939 & 1.978 \\
\hline
\end{tabular}

From Table 2, it is also observed that the inter vehicular avoidance and static obstacle avoidance have been implemented successfully during path planning.

\section{Conclusion}

In this paper, bio- inspired multi-agent system (MAS) is employed to address personal path planning problem to navigate a team of AUVs towards their destination. During path planning of a group of AUVs, the time taken to reach the desired trajectory as well as to keep the collision avoidance of AUVs are important factors. Each AUV is designated as agent connected by a communication network and assumes full communication. The agent-AUVs are identical and estimate the relative position of their neighbour AUVs while moving towards their respective destinations. The proposed multi-
AUV system constitutes of a leader AUV and three follower AUVs. The proposed MAS implement DPC algorithm to maintain coordination, safe distance among agent-AUVs and obstacle avoidance by imposing distance constraint. The agents switch from one state to another with progress in time till they reach the desired path by following the Leader AUV. The simulation results are verified that the proposed method is successful in coordinated path planning of multi- AUV system. The proposed bio-inspired multi-AUV system can be used to discover unknown under sea terrains, for the surveillance of sea-bed and to access difficult areas in underwater environment.

This is an Open Access article distributed under the terms of the Creative Commons Attribution License.

\section{References}

1. M. Panda, B. Das, B. Subudhi, and B. B. Pati, "A Comprehensive Review of Path Planning Algorithms for Autonomous Underwater Vehicles," Int. J. Autom. Comput., vol. 17, no. 3, pp. 321-352, Jun. 2020.

2. L. Durica, B. Micieta, P. Bubenik, and V. Binasova, "MANUFACTURING MULTI-AGENT SYSTEM WITH BIO-
INSPIRED TECHNIQUES: CODESA-PRIME," MM SJ, vol. 2015, no. 04, pp. 829-837, Dec. 2015.

3. B. Das, B. Subudhi, and B. B. Pati, "Formation control of underwater vehicles using Multi Agent System," Archives of Control Sciences, vol. 30, no. 2, pp. 365-384, 2020.

4. M. Panda, B. Das, and B. B. Pati, "Grey wolf optimization for global path planning of autonomous underwater vehicle," in Proceedings of 


\section{Sarada Prasanna Sahoo, Bikramaditya Das and Bibhuti Bhusan Pati/ \\ Journal of Engineering Science and Technology Review 14 (5) (2021) 173 - 179}

the Third International Conference on Advanced Informatics for Computing Research - ICAICR '19, Shimla, India, 2019, pp. 1-6.

5. B. Das, B. Subudhi, and B. B. Pati, "Employing nonlinear observer for formation control of AUVs under communication constraints," International Journal of Intelligent Unmanned Systems, vol. 3, no. 2/3, pp. 122-155, 2015 .

6. B. Das, B. Subudhi, and B. B. Pati, "Co-operative control of a team of autonomous underwater vehicles in an obstacle-rich environment," Journal of Marine Engineering and Technology, vol. 15, no. 3, pp. 135-151, 2016.

7. B. Das, B. Subudhi, and B. B. Pati, "Co-operative control coordination of a team of underwater vehicles with communication constraints," Transactions of the Institute of Measurement and Control, vol. 38, no. 4, pp. 463-481, 2016.

8. M. Panda, B. Das, and B. B. Pati, "Global path planning for multiple AUVs using GWO," Archives of Control Sciences, vol. 30, no. 1, pp. 77-100, 2020.

9. M. Panda, B. Das, and B. B. Pati, "A Hybrid Approach for Path Planning of Multiple AUVs," in Innovation in Electrical Power Engineering, Communication, and Computing Technology, Singapore, 2020, pp. 327-338.

10. S. Ul'Yanov and N. Maksimkin, "Software toolbox for analysis and design of nonlinear control systems and its application to multi-AUV path-following control," in 2017 40th International Convention on Information and Communication Technology, Electronics and Microelectronics, MIPRO 2017 - Proceedings, Jul. 2017, pp. 10321037.

11. R. Olfati-Saber, J. A. Fax, and R. M. Murray, "Consensus and cooperation in networked multi-agent systems," Proceedings of the IEEE, vol. 95, no. 1, pp. 215-233, 2007.

12. L. B. Arranz and C. C. de Wit, "Multi-agent control under communication constraints," 2008.

13. P. Szymak and T. Praczyk, "Control systems of underwater vehicles in multi-agent system of underwater inspection," in WSEAS International Conference. Proceedings. Mathematics and Computers in Science and Engineering, 2009, pp. 153-156.

14. H. Yang and F. Zhang, "Geometric formation control for autonomous underwater vehicles," in 2010 IEEE International Conference on Robotics and Automation, 2010, pp. 4288-4293.

15. D. Xue, J. Yao, J. Wang, Y. Guo, and X. Han, "Formation control of multi-agent systems with stochastic switching topology and timevarying communication delays," IET Control Theory \& Applications, vol. 7, no. 13, pp. 1689-1698, Sep. 2013.

16. Z. Hu, C. Ma, L. Zhang, A. Halme, T. Hayat, and B. Ahmad, "Formation control of impulsive networked autonomous underwater vehicles under fixed and switching topologies," Neurocomputing, vol. 147, pp. 291-298, Jan. 2015.

17. C. X. S. Viel, "Control law and state estimators design for multiagent system with reduction of communications by event-triggered approach," PhD Thesis, 2018.

18. J. Santiaguillo-Salinas and E. Aranda-Bricaire, "Time-varying Formation Tracking with Collision Avoidance for Multi-agent Systems**This work was partially supported by CONACyT, México, through scholarship holder No. 243226.," IFACPapersOnLine, vol. 50, no. 1, pp. 309-314, Jul. 2017.

19. Y. Liu, H. Yu, P. Shi, and C.-C. Lim, "Formation control and collision avoidance for a class of multi-agent systems," Journal of the Franklin Institute, vol. 356, no. 10, pp. 5395-5420, Jul. 2019.
20. Z. Zuo, M. Defoort, B. Tian, and Z. Ding, "Distributed Consensus Observer for Multi-Agent Systems With High-Order Integrator Dynamics," IEEE Trans. Automat. Contr., pp. 1-8, 2019.

21. O. Arikan, S. Chenney, and D. A. Forsyth, "Efficient multi-agent path planning," in Computer Animation and Simulation 2001, Springer, 2001, pp. 151-162.

22. K.-H. C. Wang and A. Botea, "Fast and Memory-Efficient MultiAgent Pathfinding.," in ICAPS, 2008, pp. 380-387.

23. K.-H. C. Wang and A. Botea, "MAPP: a scalable multi-agent path planning algorithm with tractability and completeness guarantees," Journal of Artificial Intelligence Research, vol. 42, pp. 55-90, 2011.

24. S. Bhattacharya, M. Likhachev, and V. Kumar, "Distributed path consensus algorithm," Technical Report MS-CIS-10-07, University of Pennsylvania, 2010.

25. S. Bhattacharya, M. Likhachev, and V. Kumar, "Multi-agent path planning with multiple tasks and distance constraints," in 2010 IEEE International Conference on Robotics and Automation, Anchorage, AK, May 2010, pp. 953-959.

26. H. Gaber, S. Amin, and A.-B. M. Salem, "A combined coordination technique for Multi-Agent Path Planning," in 2010 10th International Conference on Intelligent Systems Design and Applications, Cairo, Egypt, Nov. 2010, pp. 563-568.

27. V. R. Desaraju and J. P. How, "Decentralized path planning for multi-agent teams in complex environments using rapidly-exploring random trees," in 2011 IEEE International Conference on Robotics and Automation, Shanghai, China, May 2011, pp. 4956-4961.

28. J. Yu and S. M. LaValle, "Multi-agent Path Planning and Network Flow," in Algorithmic Foundations of Robotics X, Berlin, Heidelberg, 2013, pp. 157-173.

29. Y. Deng, P.-P. J. Beaujean, E. An, and E. Carlson, "Task Allocation and Path Planning for Collaborative Autonomous Underwater Vehicles Operating through an Underwater Acoustic Network," Journal of Robotics, vol. 2013, pp. 1-15, 2013.

30. B. de Wilde, A. ter Mors, and C. Witteveen, "Push and Rotate: a Complete Multi-agent Pathfinding Algorithm," J. Artif. Intell. Res., vol. 51, pp. 443-492, 2014.

31. F. Rossi, S. Bandyopadhyay, M. Wolf, and M. Pavone, "Review of Multi-Agent Algorithms for Collective Behavior: a Structural Taxonomy," IFAC-PapersOnLine, vol. 51, no. 12, pp. 112-117, Jan. 2018.

32. M. Chen and D. Zhu, "A Workload Balanced Algorithm for Task Assignment and Path Planning of Inhomogeneous Autonomous Underwater Vehicle System," IEEE Trans. Cogn. Dev. Syst., vol. 11, no. 4, pp. 483-493, Dec. 2019.

33. H. Ma et al., "Overview: Generalizations of multi-agent path finding to real-world scenarios," arXiv preprint arXiv:1702.05515, pp. 1-4, 2017.

34. Y. Xia, X. Na, Z. Sun, and J. Chen, "Formation control and collision avoidance for multi-agent systems based on position estimation," ISA Transactions, vol. 61, pp. 287-296, Mar. 2016.

35. B. Das, B. Subudhi, and B. B. Pati, "Adaptive sliding mode formation control of multiple underwater robots," Archives of control Sciences, vol. 24, no. 4, pp. 515-543, 2014.

36. M. Panda, B. Das, B. Subudhi, and B. B. Pati, "Adaptive fuzzy sliding mode formation controller for autonomous underwater vehicles with variable payload," International Journal of Intelligent Unmanned Systems, 2020. 